\title{
Padres y madres corresponsables. Una utopía real. María José González y Teresa Jurado Guerrero (eds.) Madrid: La Catarata, 2015
}

\author{
Sandra Dema Moreno \\ Universidad de Oviedo \\ demasandra@uniovi.es
}

Este libro es, en mi opinión, el resultado de una de las investigaciones sobre Sociología de la Familia más interesantes llevadas a cabo en los últimos años en nuestro país. Componen el equipo un potente grupo de investigadoras/es en nuestra disciplina, liderado por las editoras del libro, María José González (Universitat Pompeu Fabra) y Teresa Jurado Guerrero (Universidad Nacional de Educación a Distancia). Completan el equipo Paco Abril (Universitat de Girona), Carmen Botía Morillas (Universidad de Sevilla), Marta Domínguez Folgueras (Science Po, París), Irene Lapuerta (Universidad Pública de Navarra), Teresa Martín García (Centro Superior de Investigaciones Científicas), Jordi M. Monferrer (Universidad a Distancia de Madrid) y Marta Seiz (Centro Superior de Investigaciones Científicas).

Este grupo ha elegido un tema de investigación vital no sólo para la comprensión de nuestra sociedad, sino también para la del conjunto de países de nuestro entorno. Su objeto de estudio consiste en el análisis de la organización de la carga global de trabajo, esto es, cómo distribuyen las parejas de doble ingreso el trabajo remunerado y no remunerado en una etapa decisiva de su ciclo vital, el momento del nacimiento del/a primer/a hijo/a. Quienes nos dedicamos a los estudios de género llevamos décadas poniendo el acento en el valor social y económico del cuidado para el sostenimiento de la vida humana, señalando la tensión entre las exigencias laborales y la vida familiar y personal, e indicando las consecuencias que genera dicha tensión sobre las relaciones de género. Lo novedoso de la investigación en la que se basa este libro radica en averiguar cómo viven las parejas esta tensión en nuestro país.

Desde el punto de vista metodológico se adopta un enfoque cualitativo basado en entrevistas en profundidad a 68 parejas heterosexuales, con dos ingresos, realizadas en las ciudades de Barcelona, Madrid, Pamplona y Sevilla. Las parejas fueron entrevistadas en dos momentos: antes del nacimiento de su primera criatura y entre los 18 y 24 meses de vida de la misma. Esta metodología tiene una clara semejanza con la puesta en marcha en nuestras investigaciones sobre el análisis del dinero en las parejas de doble ingreso hace ya más de una década ${ }^{1}$. La idea de entrevistar a los dos miembros de la pareja juntos y por separado se revela especialmente útil para el estudio de las dinámicas familiares, no solo porque proporciona una enorme

1 Los hallazgos de dichas investigaciones se encuentran recogidos en diversas publicaciones, entre las que destacan: Dema Moreno, Sandra y Capitolina Díaz Martínez (2010): "Gender inequalities and the role of money in Spanish dual-income couples". European Societies 12 (1): 65-84. Dema Moreno, Sandra (2009): "Behind the Negotiations: Financial Decision-Making Processes in Spanish Dual-Income Couples". Feminist Economics 15 (1): 27-56. Dema Moreno, Sandra (2006): Una pareja, dos salarios: El dinero y las relaciones de poder en las parejas de doble ingreso. Madrid, CIS. Díaz Martínez, Capitolina; Cecilia Díaz Méndez, Sandra Dema Moreno y Marta Ibáñez Pascual (2004): Dinero, amor e individualización: Las relaciones económicas en las parejas/ familias contemporáneas. Oviedo, Editorial KRK, Colección Alternativas. 
riqueza informativa, sino también porque permite entender la forma en la que ambos miembros de la pareja interactúan.

La única duda metodológica que se suscita en el caso que nos ocupa es la secuencia empleada. En la primera ola de este estudio se realizaron entrevistas de forma individual a cada uno de los miembros de la pareja y a continuación una entrevista conjunta, con ambos integrantes simultáneamente; en la segunda ola solamente se llevaron a cabo entrevistas individuales. En nuestra investigación, por el contrario, optamos por comenzar por las entrevistas conjuntas y continuar con las individuales. Nos parece que este orden facilitó, por un lado, que las personas entrevistadas pudieran generar una narración acerca de su vida como pareja y que después cada persona pudiera expresarse sin estar condicionada por la presencia de su compañero/a y/o matizar aspectos de lo dicho en la entrevista conjunta. Si se invierte el procedimiento, no cabe este tipo de matización y si, además, en la segunda ola solo se recurre a las entrevistas individuales se pierde la riqueza de discurso y de interacción que proporciona la entrevista conjunta, dificultando en cierta medida la comparación de los relatos conjuntos antes y después del nacimiento de la criatura.

Más allá de los aspectos metodológicos, el libro se compone de seis capítulos. En el primero de ellos se sitúa el contexto socioeconómico en el que se desarrolla la investigación, que coincide precisamente con los años relativos a la crisis económica, y en los siguientes se van abordando las principales cuestiones derivadas del análisis de las entrevistas. Es interesante observar en el conjunto de la obra las diferencias entre el ideal igualitario de las parejas entrevistadas y las decisiones y acciones que van desarrollando en la práctica. Una divergencia que deriva en gran parte de los condicionamientos de tipo económico, cultural e institucional, que se reproducen y retroalimentan en el interior de los hogares y que dificultan la construcción de relaciones igualitarias. Un resultado de nuevo similar al obtenido en nuestras investigaciones sobre las parejas y el dinero. Este paralelismo, en mi opinión, refuerza los hallazgos de ambos equipos que, utilizando un método parecido, aunque el objeto de estudio no sea exactamente el mismo, fueron capaces de detectar dinámicas de (des)igualdad de género semejantes.

El análisis de las entrevistas constituye la parte sustancial de este libro y, como sucede con las investigaciones cualitativas, los relatos de las personas entrevistadas tienen un extraordinario potencial a la hora de mostrar la realidad en la que viven las parejas y los dilemas a los que se enfrentan, pero sobre todo en estos capítulos se evidencia la capacidad analítica de sus autores/ as. El material cualitativo permite indagar acerca del significado que las mujeres y los varones a punto de tener descendencia otorgan a la maternidad y a la paternidad, así como los cambios que experimentan al nacer el primer bebé. En los capítulos 2 y 3 se explican asimismo las estrategias de cuidado que adoptan las parejas al nacer la criatura y cómo el acceso desigual a los permisos de maternidad y paternidad a los que tienen derecho mujeres y hombres en nuestro país favorece que las primeras asuman la responsabilidad principal del cuidado. Se estudia también el vínculo entre maternidad/paternidad y empleo y en qué medida el nacimiento del primer/a hijo/a conlleva o no cambios tanto en la percepción del empleo, como en las estrategias que adoptan padres y madres a la hora de incorporarse al mismo tras la finalización de los permisos.

El equipo investigador es capaz de identificar diferentes modelos de maternidad y paternidad que conviven actualmente en nuestro país, desde los más tradicionales, pasando por aquellos en los que aparecen tensiones entre el deseo y la realidad, pero también aquellos otros que se salen de la norma y que, a pesar de los innumerables condicionamientos sociales, logran funcionar de forma corresponsable e implicar a ambos progenitores en el cuidado de hijas e hijos. Este modelo, en el que sus integrantes tratan de establecer formas de organización tendentes a la igualdad, es objeto de estudio más detallado en los capítulos finales del libro. En el capítulo 4 se analiza la satisfacción de estos padres y madres a la hora de conciliar empleo y cuidados tras el nacimiento del bebé, con el fin de identificar si son capaces de 
cumplir 0 no sus expectativas igualitarias en un contexto institucional que, como decíamos anteriormente, no favorece la igualdad. En el capítulo 5 se explican las formas de organización del trabajo doméstico antes y después del nacimiento de la criatura, para averiguar si dicho acontecimiento promueve o no la adopción de formas de reparto más tradicionales. Y, por último, en el capítulo 6 se relatan detalladamente los casos de cinco parejas que el equipo investigador entiende que son explicativos de los diferentes procesos analizados a lo largo del libro.

Confío en que esta rápida descripción tanto de la metodología como de los contenidos de la obra haya podido despertar la curiosidad de lectores y lectoras acerca de una investigación que aporta información extremadamente detallada acerca de cómo funcionan las dinámicas familiares y de género en relación con la fecundidad y los cuidados. El diagnóstico que nos proporciona se resume en unas conclusiones palpables y demoledoras. Por hacer referencia sólo a algunas de ellas: la crisis ha supuesto una reducción de la ya de por sí baja fecundidad española y un nuevo retraso de la edad materna al dar a luz a su primer/a hijo/a, mostrando la influencia de la incertidumbre económica y la precariedad en las decisiones reproductivas. Asimismo, el cuidado y el reparto equitativo del mismo en las parejas de doble ingreso se revela como una cuestión extremadamente complicada, sobre todo si no se dispone de una red de apoyo familiar y/o de recursos suficientes para externalizar al menos una parte de dicho trabajo. La investigación constata que el nacimiento de las criaturas aumenta las tareas de cuidados y refuerza la división sexual del trabajo, en detrimento de las mujeres. $Y$ finalmente, se muestra cómo aquellas parejas que logran funcionar de manera igualitaria lo hacen con un esfuerzo tremendo, por una clara vocación y compromiso igualitario, podríamos decir que a pesar 0 incluso en contra del sistema.

Este libro pone su punto final precisamente cuando querríamos que continuara. Si el modelo de reparto del trabajo remunerado y no remunerado que tenemos es insostenible, como confirma no solo esta investigación sino otras muchas, la pregunta obligada que debemos hacernos es cómo organizar socialmente dicho reparto de forma más equitativa. En el libro se mencionan algunas iniciativas, como la demanda de permisos iguales e intransferibles para hombres y mujeres, y en las conclusiones se apunta tímidamente a la necesidad de reducción de la jornada laboral a 35 horas.

Creo que efectivamente las políticas públicas deben ir en esa línea, pero en mi opinión, urge abrir un debate más amplio sobre la necesidad de reformular los derechos económicos y sociales necesarios para garantizar un modelo de sociedad y un ejercicio igualitario de la ciudadanía por parte de mujeres y varones. Como ya planteamos en otras ocasiones y en la línea de lo que también señalan conocidas economistas feministas, como Cristina Carrasco, Lina Gálvez o Amaia Pérez Orozco, entre otras, tales derechos pasan por el reparto equitativo de la carga global de trabajo, remunerado y no remunerado, más acuciante si cabe debido a la crisis. El cuidado, no solo de los/ ss niños/as, sino del conjunto de personas que formamos parte de la sociedad, debería estar garantizado y ser considerado no una responsabilidad individual y/o familiar, sino un derecho fundamental de ciudadanía. La diferencia es muy importante: si entendemos el cuidado como un derecho lo sacamos del ámbito del hogar, deja de ser un problema privado de las familias, y fundamentalmente de las mujeres, para formar parte de la agenda política. El cuidado entendido como derecho nos obliga a debatir acerca de las fórmulas para asegurar su provisión a toda la ciudadanía, no solo a las criaturas, sino a lo largo de todo el ciclo vital de las personas, sobre todo en la vejez. Un reto especialmente importante en una sociedad cada vez envejecida, como la nuestra.

Pero, además, como bien nos muestra esta investigación trabajo remunerado y no remunerado están claramente relacionados. Si ponemos a las personas y su bienestar en el centro de la agenda política, el empleo también altera su significado. Nuestras normas jurídicas sancionan el derecho al trabajo, sin embargo, en un país con un desempleo estructural como el que padecemos desde hace décadas este derecho cada vez está 
más vacío de contenido. A esta circunstancia se añade que su ejercicio no es igualitario para hombres y mujeres, porque sobre estas últimas recae en buena medida el trabajo de cuidado. El modelo de jornada laboral de 40 o más horas está caduco, se pensó sobre la base de que el varón trabajaba de forma remunerada, mientras que la mujer se hacía cargo del trabajo doméstico. Pero como este libro constata, cada vez existen menos familias con un ama de casa dedicada al trabajo doméstico a tiempo completo y cada vez menos familias pueden permitirse vivir con un solo salario. Tampoco se trata de fomentar el empleo a tiempo parcial, como hacen algunos países de nuestro entorno, porque esas medidas provocan que quienes ocupan tales trabajos, en su mayoría mujeres, ganen menos, coticen menos y en el futuro tengan pensiones más bajas, al tiempo que se promueve que sean ellas quienes asuman la mayor parte del trabajo doméstico.

De lo que se trata es de un cambio de modelo que permita repartir en condiciones de igualdad tanto el trabajo remunerado como el no remunerado. Si repartimos el empleo que se genera en nuestro país de forma equilibrada entre todas las personas en edad de trabajar podríamos reducir la jornada laboral a 20-25 horas semanales. Una medida como esta nos permitiría garantizar el cuidado y el bienestar de la ciudadanía y, al mismo tiempo, acabar con el desempleo estructural, que aboca a millones de personas a no poder desarrollar su potencial y a ser dependientes de otras. Puede que la propuesta suene utópica, pero creo que se dan las condiciones sociales, económicas y tecnológicas para que esta utopía se convierta en real, lo que no está tan claro es que los agentes sociales y políticos estén a la altura de una propuesta de este tipo?

Un tercer elemento del debate es el de la protección social. Especialmente en tiempos de crisis, las sociedades deben de ser capaces de proteger a su población, puesto que supone la mejor forma de evitar la vulnerabilidad, a la vez que constituye un mecanismo que permite controlar la desigualdad y generar empleo. Tenemos un sistema de seguridad social pública que hay que replantear para garantizar la igualdad entre hombres y mujeres. Si aspiramos a un modelo igualitario, el acceso a las prestaciones y a los servicios que presta el Estado debe ser universal, de manera que cada persona por el hecho de serlo, independientemente de su forma de convivencia $y / 0$ de su vinculación laboral, pueda ejercer los derechos que le permitan desarrollar una vida digna.

Muchas colegas, sobre todo economistas, llevan décadas denunciando sin demasiado éxito que el modelo económico, laboral, de relaciones sociales y de género estaba en quiebra. La crisis les ha dado la razón. En este libro hemos podido conocer de primera mano los efectos que dicho modelo ha ocasionado sobre las familias españolas y sus decisiones reproductivas. El reto ahora está en aprovechar todo este acervo de conocimiento que producimos desde el ámbito académico para construir una sociedad más igualitaria, solidaria y sostenible, una utopía que ojalá pronto se convierta en realidad.

2 Para profundizar en el debate sobre el reparto de la jornada de trabajo resulta de interés el estudio: New Economics Foundation (2012): 21 horas. Una semana laboral más corta para prosperar en el siglo xx. BarceIona, Icaria. 